
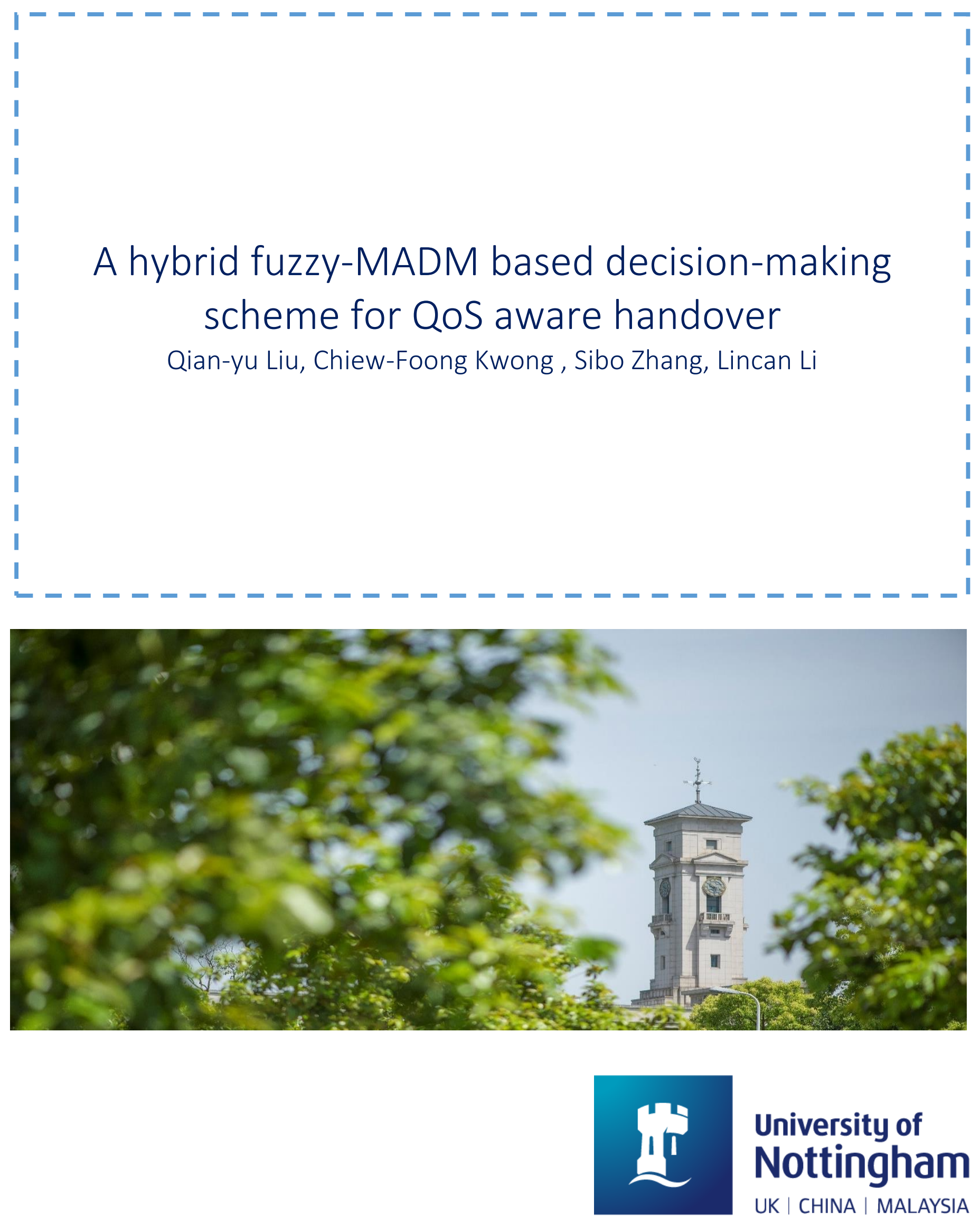

University of Nottingham 
International Doctoral Innovation Centre, University of Nottingham Ningbo China, Ningbo, China

Department of Electrical and Electronic Engineering, University of Nottingham Ningbo China, Ningbo, China

First published 2018

Copyright 2018 Qian-yu Liu, Chiew-Foong Kwong , Sibo Zhang, Lincan Li

This work is made available under the terms of the Creative Commons Attribution 4.0 International License: http://creativecommons.org/licenses/by/4.0

The work is licenced to the University of Nottingham Ningbo China under the Global University Publication Licence:

https://www.nottingham.edu.cn/en/library/documents/researchsupport/global-university-publications-licence.pdf 


\title{
A hybrid fuzzy-MADM based decision-making scheme for QoS aware handover Qian-yu Liu' ${ }^{1}$, Chiew-Foong Kwong ${ }^{2 *}$, Sibo Zhang', Lincan Li ${ }^{2}$
}

\author{
${ }^{I}$ International Doctoral Innovation Centre, University of Nottingham Ningbo China, Ningbo, China \\ ${ }^{2}$ Department of Electrical and Electronic Engineering, University of Nottingham Ningbo China, Ningbo, China \\ *Chiew-Foong.Kwong@nottingham.edu.cn
}

Keywords: Handover, Fuzzy Logic, Multiple Attribute Decision Making, Quality of Service

\begin{abstract}
The fifth-generation communications system (5G) will commercialize at 2020 in order to satisfy the increasing demands on data rate and also to enable the internet of things (IoT). One of the most challenging issues in 5G communications network is to provide provisioning quality of service (QoS) while maintaining seamless mobility for user equipment (UE). This paper proposes a QoS-aware handover algorithm based on fuzzy-TOPSIS to trigger and achieve the optimal cell selection. The proposed algorithm integrates both advantages of fuzzy logic and technique for order preference by similarity to an ideal solution (TOPSIS). The weights value of network attributes is first calculated by Entropy and the fuzzy-TOPSIS algorithm are then applied to rank each access networks. This QoS-aware algorithm is able to achieve the optimal Mean Option Score (MOS) for UE by considering QoS related parameters such as network jitter and packet loss ratio. The simulation results indicate that the proposed algorithm can guarantee good QoS while maintaining number of handover at a low level.
\end{abstract}

\section{Introduction}

The fifth-generation communications system will launch in 2020. One of the main features of $5 \mathrm{G}$ is to deploy high dense small cell in order to provide ubiquitous connection and provisioning QoS for UE. Owing to this feature and high mobility of future $5 \mathrm{G}$ application scenario, there are serious of mobility related issues could be raised i.e. ping-pong handover, handover failures, frequent/unnecessary handover etc. To avoid these effects and ensure good QoS for UE, the handover of UE need to be triggered at the exact right timing and then switch to a base station (BS) with the highest QoS.

The current handover in the LTE system is based on the X2 interface which consists of three stages: the preparation, execution and completion stage [1]. In the preparation stage, the UE will collect handover related information such as reference signal received power (RSRP), received signal strength indication (RSSI) from neighbouring BSs and sends to its serving BSs. Based on this collected information, the serving BSs of UE will decide when to trigger handover and also select one neighbouring BSs as handover target. At the next stage, the serving BS of UE will execute the handover process and switch its connection to targeted BS by adopting a hard HO mechanism. The hard handover operates with the concept of "break before make" and that can result in a short link break before connection of UE switch to the target BS. The handover process ends with the updates of user plane as well as the resources releasing of serving BS. Base on that, if the timing of triggering is too early or too late or the selected target BS is not the most suitable selection, the abnormal handover could occur.

To mitigate the effects of handover triggering, some researchers proposed two parameters which named as handover margin (HOM) and time to trigger (TTT) for triggering. This approach allows a time-interval within handovers and hence to avoid unnecessary handover occurs. However, the value of these two parameters needs to adjust at the different scenario, which need to conduct extensive measuring campaign and data analysis. Fuzzy logic based handover algorithm is an effective tool to conduct multivariate analysis and process uncertainty data within environments which can hence enable both timely and flexibility handover [2], [3]. However, when the system considers more than three criteria as the input of fuzzy logic, the whole fuzzy inference system will become complicated and difficult to define a proper fuzzy rule.

On the other hand, the conventional $\mathrm{HO}$ algorithm use only a single metric i.e. RSSI, RSRP as decision criteria for triggering and target BS selection. As such, the handover process is easily affected by interference and cause UE frequently switch connection between BSs which known as ping-pong effect. In addition, the single metric-based handover cannot satisfy the actual demand of future mobile users. To consider more than one attributes and criteria into BSs selection, one of approach is to adopt multi-attributes decision making (MADM) scheme as shown in [4]-[6]. MADM is a reliable mathematical tool to handle multiple conflicting attributes in a decision-making problem. Thus, the MADM can support serving BS of UE to select the most suitable access networks from various candidates in relation to multiple criteria. In general, there are four types of MADM scheme are widely used, i.e. simple additive weighting (SAW) [7]-[9], techniques for order preference by similarity for an ideal solution (TOPSIS) [10][13], analytic hierarchy process (AHP) [14], [15] and Grey relational analysis (GRA). However, these MADM algorithms have several of inherent drawbacks. Firstly, the performance of MADM is highly relying on its weighting method. Normally, the subjective weight value of each criterion is defined by human experience. As the mobile operator may not 
have full knowledge and experience on related criteria, and hence the heavy reliance on human experience is unreliable. Besides that, the conventional MADM cannot process uncertain and imprecise information within decision-making problem conclusively. When collected data of UE with minor deviation, such as unpredictable radio signal fluctuations, the selected target BS are usually not optimal.

Moreover, to select a suitable BS as handover target while maintaining good QoS is remained as a significant challenge for the mobile operators. The conventional QoS based handover algorithm will frequently switch connection between BSs in order to maintain good MOS. To overcome the drawbacks in fuzzy logic, MADM and QoS aware handover algorithm, this paper presents a novel QoS-aware handover algorithm which integrates both advantages of fuzzy logic and TOPSIS. The proposed algorithm can trigger $\mathrm{HO}$ based on the overall performance of serving BSs and each neighbouring BSs. Apart from that, this algorithm can process uncertain data and consider more than one metric such as jitter, packet loss and signal to noise ratio (SNR) to select handover target in order to provide good QoS to mobile user. In addition, the entropy is implemented to obtain objective weight value of each criterion, which can eliminate the subjective error within a human decision.

The rest of this paper is organised as follow: Section 2 shows the comprehensive methodology of fuzzy-MADM handover scheme. And the proposed algorithm will be tested in a simulation environment and compared with the conventional handover algorithm in section 3 . The conclusion and future work will be finally given at section 4 .

\section{Methodology}

The procedure of fuzzy-TOPSIS handover algorithm is shown in Fig 1. In the proposed algorithm, the fuzzy logic is first implemented to convert handover criteria into a crispy format as the input for TOPSIS. The TOPSIS act as the main decisionmaking engine in the algorithm.

While moving, UE will frequently collect handover and QoS related parameters for each neighbouring BS i.e. Packet loss, jitter, SNR, RSSI and report to its serving BS. The BS will form a decision matrix $\boldsymbol{D M}$ for each candidate $\mathrm{BS}$ in relation to its criteria as shown in (1).

$$
\begin{aligned}
& \begin{array}{lllllll}
C_{1} & C_{2} & C_{3} & \ldots & \ldots & C_{n}
\end{array} \\
& D M=\begin{array}{c|cccccc}
A_{1} & x_{11} & x_{12} & x_{13} & \ldots & \ldots & x_{1 n} \\
A_{2} & x_{21} & x_{22} & x_{23} & \ldots & \ldots & x_{2 n} \\
\vdots & \vdots & \vdots & \vdots & & & \vdots \\
\vdots & \vdots & \vdots & \vdots & & & \vdots \\
A_{m} & x_{m 1} & x_{m 2} & x_{m 3} & \ldots & \ldots & x_{m n}
\end{array} \mid
\end{aligned}
$$

In decision matric $\boldsymbol{D} \boldsymbol{M}$, each element $x_{i j}$ represents the value of the attributes $A_{i}$ (i from 1 to $m$ ) for the handover criteria $C_{j}$ ( $\mathrm{j}$ from 1 to $\mathrm{n}$ ). For example, $\mathrm{A}_{1}$ is $\mathrm{BS}_{1}$ with $\mathrm{n}$ handover criteria from $\mathrm{x}_{11}$ to $\mathrm{x}_{1 \mathrm{n}}$.

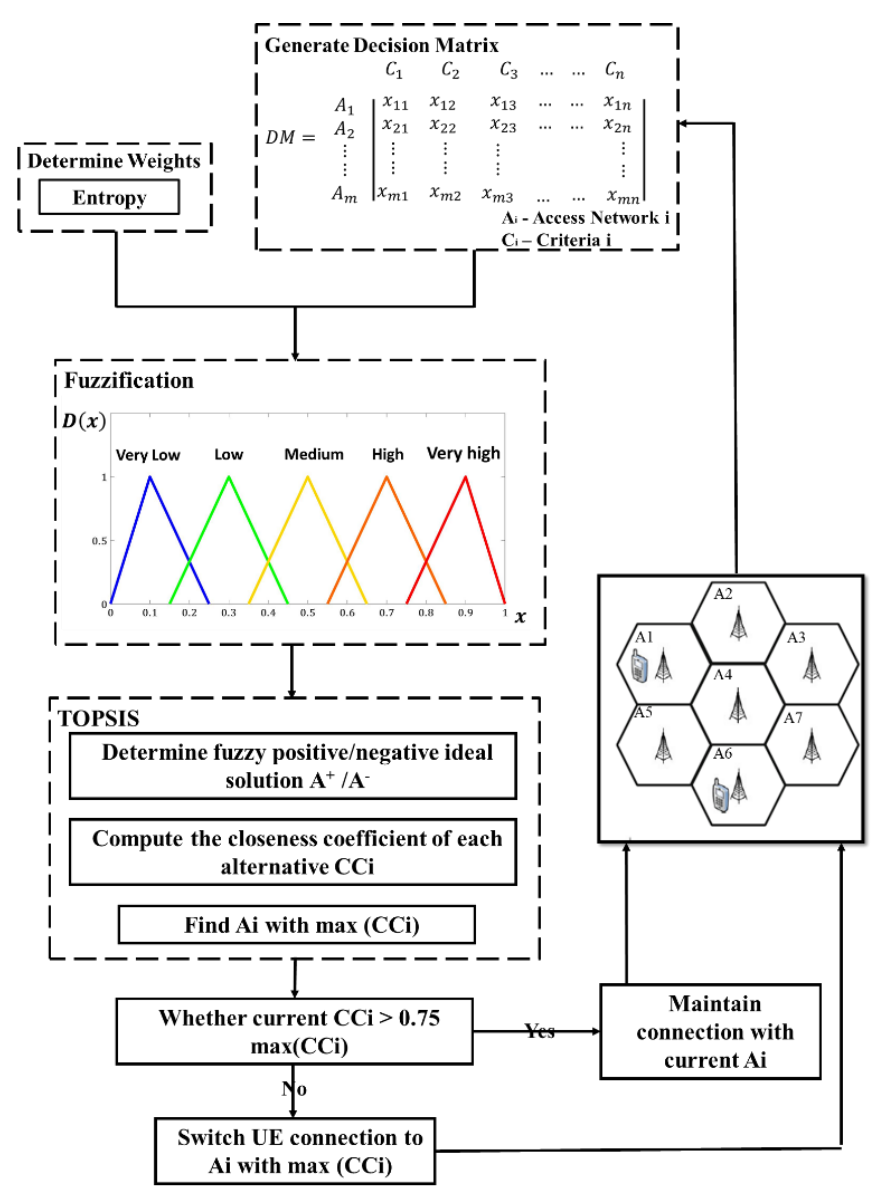

Fig. 1 Procedure of Fuzzy-TOPSIS handover algorithm

In the next stage, each element in DM will be normalized into the dimensionless format by Min-Max scaling approach as shown in (2) and (3) for both benefit and cost handover criteria.

$$
\begin{aligned}
& Z_{i j}=\frac{\left[x_{i j}-\min \left\{x_{i j}\right\}\right]}{\left[\max \left\{x_{i j}\right\}-\min \left\{x_{i j}\right\}\right]} \\
& Z_{i j}=\frac{\left[\max \left\{x_{i j}\right\}-x_{i j}\right]}{\left[\max \left\{x_{i j}\right\}-\min \left\{x_{i j}\right\}\right]}
\end{aligned}
$$

After this stage, the normalized decision matrix can be hence obtained. An objective weighting approach- entropy method is then implemented to obtain weight value for each criterion in order to eliminate human subjective error. The element in normalized decision matrix and its corresponding weight value will be converted to crispy format by mapping into a triangular fuzzy membership function as indicated in (4) and Fig.2. This stage is known as fuzzification in fuzzy logic. The normalized matrix and weight value are hence converted into the normalized fuzzy decision matrix $\widetilde{\mathrm{DM}}$ and fuzzy weight array $\widetilde{\mathrm{W}}$ as (5) and (6).

$$
D(x)=\left\{\begin{array}{lr}
0 & x \leq a_{1} \\
\frac{x-a_{1}}{a_{2}-a_{1}} & a_{1}<x \leq a_{2} \\
\frac{a_{3}-x}{a_{3}-a_{2}} & a_{2}<x \leq a_{3} \\
1 & x>a_{3}
\end{array}\right.
$$




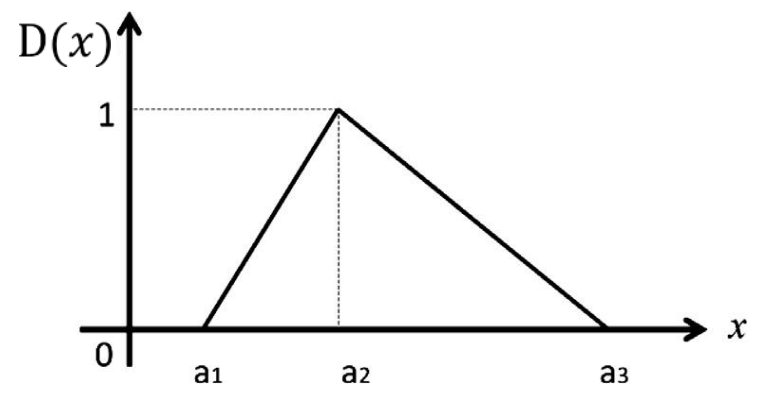

Fig. 2 Triangular Fuzzy number D(x)

$$
\begin{aligned}
& \begin{array}{lllllll}
C_{1} & C_{2} & C_{3} & \ldots & \ldots & C_{n}
\end{array} \\
& \widetilde{D M}=\begin{array}{c|cccccc}
A_{1} & \tilde{x}_{11} & \tilde{x}_{12} & \tilde{x}_{13} & \ldots & \ldots & \tilde{x}_{1 n} \\
A_{2} & \tilde{x}_{21} & \tilde{x}_{22} & \tilde{x}_{23} & \ldots & \ldots & \tilde{x}_{2 n} \\
\vdots & \vdots & \vdots & \vdots & & & \vdots \\
\vdots & \vdots & \vdots & \vdots & & & \vdots \\
A_{m} & \tilde{x}_{m 1} & \tilde{x}_{m 2} & \tilde{x}_{m 3} & \ldots & \ldots & \tilde{x}_{m n}
\end{array} \mid \\
& \widetilde{W}=\left[\widetilde{w_{1}}, \widetilde{w_{2}} \ldots \ldots, \widetilde{w_{n}}\right]
\end{aligned}
$$

It the normalized fuzzy matrix $\widetilde{\mathrm{DM}}, \widetilde{x_{l j}}=\left(a_{i j}, b_{i j}, c_{i j}\right)$ represents the fuzzy membership function of jth handover criteria of ith candidate BS; the degree of importance for each handover criteria are represented by $\widetilde{w_{J}}=\left(a_{j 1}, b_{j 2}, c_{j 3}\right)$. Afterwards, the $\widetilde{\mathrm{DM}}$ will multiply with $\widetilde{\mathrm{W}}$ to calculate weighted and normalized fuzzy decision matrix $\widetilde{V}$ as,

$$
\begin{aligned}
\tilde{V} & =\left|\begin{array}{cccccc}
\tilde{v}_{11} & \tilde{v}_{12} & \tilde{v}_{13} & \ldots & \ldots & \tilde{v}_{1 n} \\
\tilde{v}_{21} & \tilde{v}_{22} & \tilde{v}_{23} & \ldots & \ldots & \tilde{v}_{2 n} \\
\vdots & \vdots & \vdots & & & \vdots \\
\vdots & \vdots & \vdots & & & \vdots \\
\tilde{v}_{m 1} & \tilde{v}_{m 2} & \tilde{v}_{m 3} & \ldots & \ldots & \tilde{v}_{m n}
\end{array}\right| \\
& =\left|\begin{array}{ccccccc}
\widetilde{w}_{1} \tilde{x}_{11} & \widetilde{w}_{2} \tilde{x}_{12} & \widetilde{w}_{3} \tilde{x}_{13} & \ldots & \ldots & \widetilde{w}_{n} \tilde{x}_{1 n} \\
\widetilde{w}_{1} \tilde{x}_{21} & \widetilde{w}_{2} \tilde{x}_{22} & \widetilde{w}_{3} \tilde{x}_{23} & \ldots & \ldots & \widetilde{w}_{n} \tilde{x}_{2 n} \\
\vdots & \vdots & \vdots & & & \vdots \\
\vdots & \vdots & \vdots & & & \vdots \\
\widetilde{w}_{1} \tilde{x}_{m 1} & \widetilde{w}_{2} \tilde{x}_{m 2} & \widetilde{w}_{3} \tilde{x}_{m 3} & \ldots & \ldots & \widetilde{w}_{n} \tilde{x}_{m n}
\end{array}\right|
\end{aligned}
$$

Based on $\tilde{V}$, the following stage is to obtain the fuzzy positive ideal solution $\left(A^{*}\right)$ and fuzzy negative ideal solution $\left(A^{-}\right)$by (8) and (9),

$$
\begin{aligned}
& A^{+}=\widetilde{V}_{J}^{+}(j=1,2, \ldots, m) \quad \text { where } \widetilde{V}_{J}^{+}=\max _{i} \widetilde{V_{l J}} \\
& A^{-}=\widetilde{V}_{J}^{-}(j=1,2, \ldots, m) \quad \text { where } \widetilde{V}_{J}^{-}=\min _{i} \widetilde{V_{l J}}
\end{aligned}
$$

From $A^{*}$ and $A^{-}$, the closeness between each candidate BSs to both fuzzy positive and negative ideal solution can be calculated by $(10)-(12)$,

$$
\begin{aligned}
& d_{i}^{+}=\sum_{j=1}^{n} d\left(\widetilde{V_{l \jmath}}, \widetilde{V}_{J}^{+}\right) \\
& d_{i}^{-}=\sum_{j=1}^{n} d\left(\widetilde{V_{l \jmath}}, \widetilde{V}_{J}^{-}\right)
\end{aligned}
$$

\begin{tabular}{l|l}
\multicolumn{2}{c}{ Table 1 Simulation parameters } \\
Parameters & Specification \\
\hline BS transmitted power: & $30 \sim 35 \mathrm{dBm}$ \\
Carrier frequency: & $1.5 \sim 2 \mathrm{GHz}$ \\
Duration of simulation & $36000 \mathrm{~s}$ \\
Mobility model & Random direction \\
Number of BSs & 16 \\
The distance between each BS & $1800 \mathrm{~m}$ \\
Number of UE & Single UE \\
UE speed & $120 \mathrm{~km} / \mathrm{h}$ \\
Propagation model: & Cost-Hata model \\
Type of Noise & AWGN
\end{tabular}

$d(\tilde{a}, \tilde{b})=\sqrt{\frac{1}{3}\left[\left(a_{1}-b_{1}\right)^{2}+\left(a_{2}-b_{2}\right)^{2}+\left(a_{3}-b_{3}\right)^{2}\right]}$

Finally, the highest closeness coefficient $\mathrm{CC}_{\mathrm{i}}$ of each candidate BSs are calculated by (13). The BSs with the highest $\mathrm{CC}_{\mathrm{i}}$ is selected as the optimal BS,

$$
C C_{i}=\frac{d_{i}^{-}}{d_{i}^{+}+d_{i}^{-}}
$$

When found the optimal $\mathrm{BS}_{\mathrm{n}}$ with the highest $\mathrm{CC}_{\mathrm{n}}$, the system will compare $\mathrm{CC}_{\mathrm{i}}$ of the serving $\mathrm{BS}_{\mathrm{i}}$ with $\mathrm{CC}_{\mathrm{n}}$ of optimal $\mathrm{BS}_{\mathrm{n}}$. Only if when $\mathrm{CC}_{\mathrm{n}}$ higher than 1.33 times of $\mathrm{CC}_{\mathrm{i}}$, the handover will be triggered. And then the connection of UE will handover to selected $\mathrm{BS}_{\mathrm{n}}$. Otherwise, the system will remain the connection of UE with current serving BS until meeting the trigger condition.

\section{Performance Evaluation}

\subsection{Simulation environment}

A simulation environment was built with MATLAB to validate the effectiveness of the proposed algorithm. The related parameter for the simulation environment is indicated in Table 1 . There are 16 BSs are deployed in a $6000 \mathrm{~m} * 6000 \mathrm{~m}$ scenario, and the distance between each of them is $1800 \mathrm{~m}$. The cost-hata model is used as the propagation model for each BSs. The Additive white Gaussian noise (AWGN) is added to the transmitted signal. A single UE will randomly move at this scenario with a constant speed at $120 \mathrm{~km} / \mathrm{h}$.

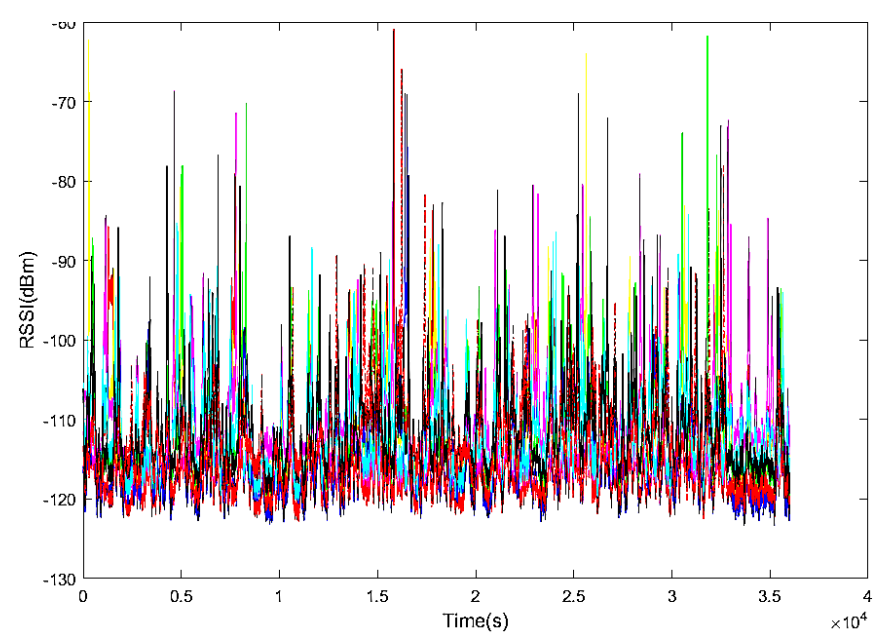

Fig. 3 RSSI of UE from each BSs 


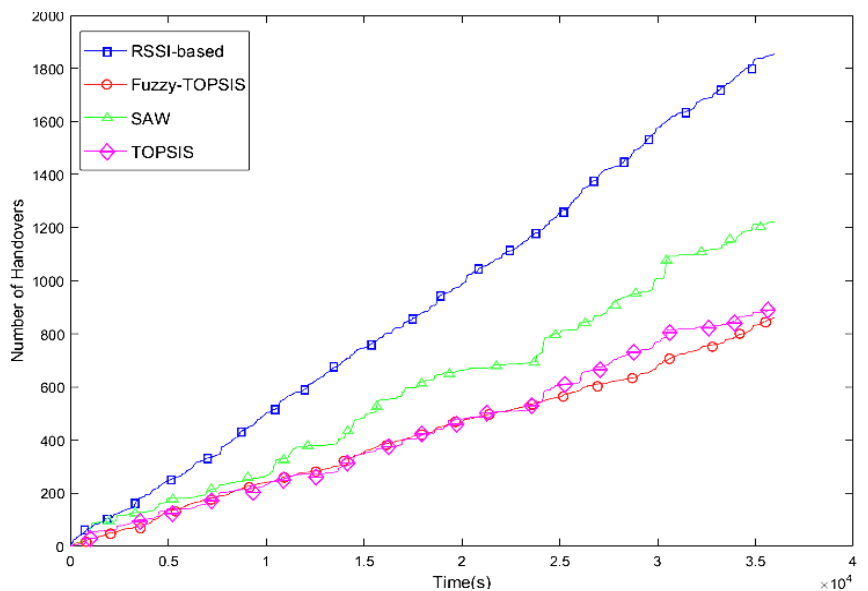

Fig. 4 Number of handovers from each method

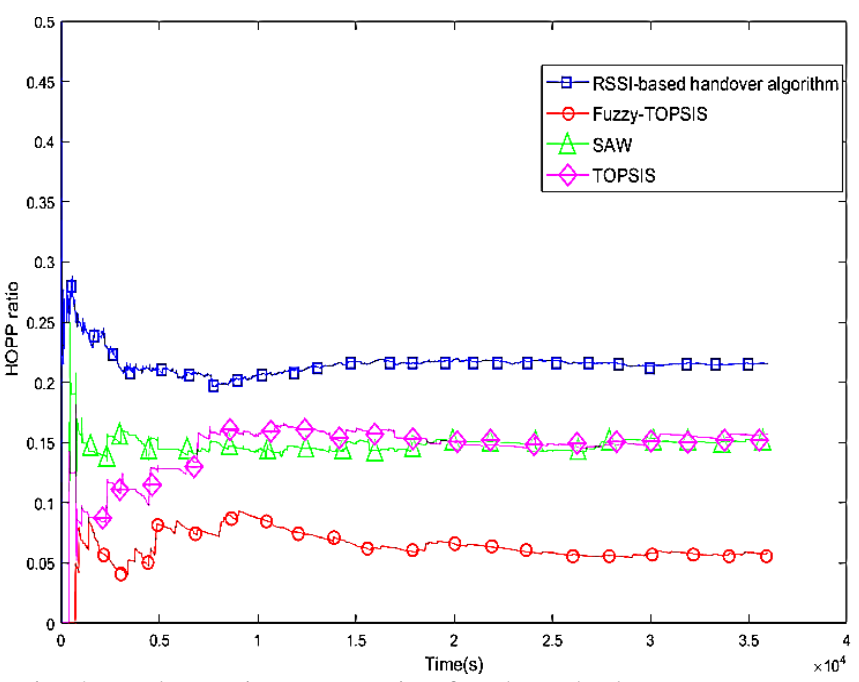

Fig. 4 Handover ping-pong ratio of each method

The RSSI, SNR, jitter and packet loss ratio are used as handover criteria in this paper. The number of handovers, ping-ping handover ratio and MOS are used as performance indicators to evaluate the performance of the proposed algorithm. The conventional RSSI-based handover algorithm, conventional SAW and TOPSIS based handover algorithm are used to compare with the proposed scheme. The ping-pong handover in this paper is defined as when UE handed back to the same serving BS within 10s. The handover ping-pong (HOPP) ratio is calculated as (14),

$$
\text { HOPP ratio }(\%)=\frac{\text { Number of Pingpong hadnover }}{\text { Number of handover }}
$$

The grade scale of MOS is separated from 5 (best) to 1 (worst). The value of MOS can be determined from either a subjective approach based on user evaluation or objective approach based on measurement of network parameters such as jitter and packet loss ratio. This paper adopts an objective approach - an improved E-Model to obtain MOS as proposed in [16].

\subsection{Results and Discussion}

Fig 3 shows the RSSI of UE from each BSs and simulation results are shown in Fig 4 - Fig 6. As presented in Fig 4, the conventional RSSI based handover algorithm has the highest number of handovers. The RSSI is fluctuating dues to the

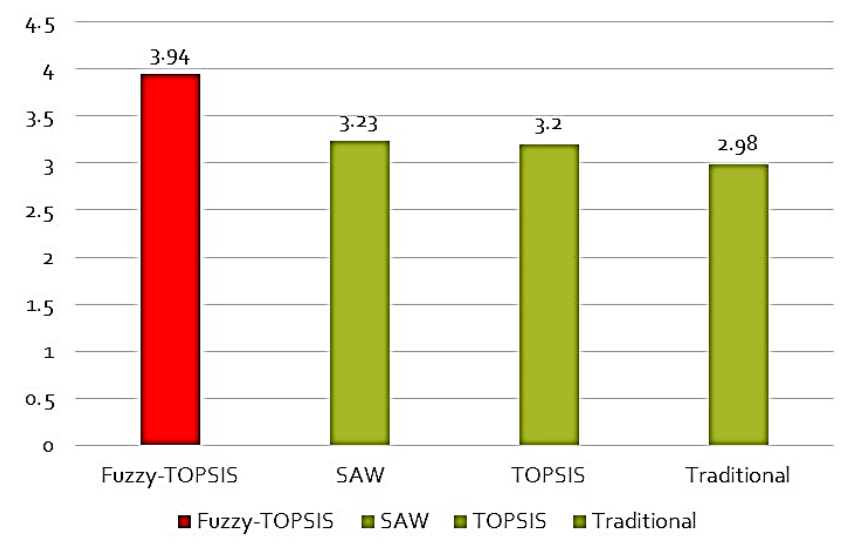

Fig. 6 Average MOS value of each method

existing of interference. If only consider RSSI as handover criteria, the UE will frequently switch to BS with higher RSSI and results ping-pong and unnecessary handover. Due to this reason, the RSSI-based handover algorithm also with the highest HOPP ratio as indicated in Fig 5. On the other hand, the proposed fuzzy-TOPSIS based algorithm has a similar number of handover with conventional TOPSIS. However, the HOPP ratio of conventional TOPSIS and SAW is almost three times higher than fuzzy-TOPSIS approach. According to Fig 6 , the proposed QoS aware algorithm can achieve the highest MOS value among all conventional approach. The conventional SAW and TOPSIS have almost the same MOS level, while the conventional RSSI-based handover algorithm with the lowest MOS level. Based on that, the proposed algorithm can not only guarantee good QoS for the user but also maintain number of handovers and PPHO ratio at a low level.

As shown from the simulation result, the conventional RSSIbased handover algorithm has the worst overall performance because it only considers single-metric as decision criteria. The SAW and TOPSIS have almost the same performance. As mention in the previous section, the performance of the conventional MADM approach highly relies on its weight method. These two MADM approaches adopt the same weighting method, and thus the overall performance of them are similar. The proposed fuzzy-TOPSIS based handover algorithm has the best performance among these methods, which showed that the involvement of fuzzification can minimize the effect of the uncertain weight value and inaccurate information. In the meantime, the TOPSIS in fuzzyTOPSIS algorithm can select the optimal candidate BS effectively.

\section{Conclusion}

This paper proposes a hybrid fuzzy-TOPSIS based QoS aware algorithm which integrates both advantages of fuzzy logic and TOPSIS. The proposed algorithm is able to trigger the handover process based on the overall performance of each BS. On the other hand, this algorithm can select the optimal BS with good QoS by considering multiple handover criteria such as RSSI, SNR, jitter and packet loss ratio. The simulation results showed that the proposed algorithm can provide good QoS for the mobile user and minimize unnecessary/frequency handover. It also outperforms conventional MADM algorithm 
i.e. SAW, TOPSIS and conventional RSSI-based algorithm. In the future research, the proposed algorithm will be fully tested in different application scenario and applied into heterogeneous communication environment.

\section{Acknowledgements}

The author acknowledges the financial support from the International Doctoral Innovation Centre, Ningbo Education Bureau, Ningbo Science and Technology Bureau, and the University of Nottingham. This work was also supported by Ningbo Natural Science Programme, project code 2018A610095.

\section{References}

[1] 3GPP, "Overview of $\{3$ GPP $\}$ release 8, v.0.1.1," 2010.

[2] M. Saeed, H. Kamal, and M. El-Ghoneimy, "A new fuzzy logic technique for handover parameters optimization in LTE," in 2016 28th International Conference on Microelectronics (ICM), 2016, pp. 53-56.

[3] M. Saeed, M. El-Ghoneimy, and H. Kamal, "An enhanced fuzzy logic optimization technique based on user mobility for LTE handover," in National Radio Science Conference, NRSC, Proceedings, 2017, no. Nrsc, pp. 230-237.

[4] B. R. Chandavarkar and R. M. R. Guddeti, "Simplified and improved multiple attributes alternate ranking method for vertical handover decision in heterogeneous wireless networks," Comput. Commun., vol. 83, pp. 81-97, 2016.

[5] M. Lahby, S. Baghla, and A. Sekkaki, "Survey and comparison of MADM methods for network selection access in heterogeneous networks," 2015 7th Int. Conf. New Technol. Mobil. Secur. - Proc. NTMS 2015 Conf. Work., 2015.

[6] Manisha and N. P. Singh, "Optimal network selection using MADM algorithms," in 2015 2nd International Conference on Recent Advances in Engineering and Computational Sciences, RAECS 2015, 2016, no. December, pp. 1-6.

[7] C. H. F. Dos Santos, M. P. S. De Lima, F. S. Dantas Silva, and A. Neto, "Performance evaluation of multiple attribute mobility decision models: A QoE-efficiency perspective," in International Conference on Wireless and Mobile Computing, Networking and Communications, 2017, vol. 2017-Octob.

[8] N. Omheni, F. Zarai, M. S. Obaidat, I. Smaoui, and L. Kamoun, "A MIH-based approach for best network selection in heterogeneous wireless networks," J. Syst. Softw., vol. 92, no. 1, pp. 143-156, 2014.

[9] A. Habbal, S. I. Goudar, and S. Hassan, "Context-Aware Radio Access Technology Selection in 5G Ultra Dense Networks," IEEE Access, vol. 5, no. Mmc, pp. 6636-6648, 2017.

[10] M. Alhabo and L. Zhang, "Multi-criteria handover using modified weighted TOPSIS methods for heterogeneous networks," IEEE Access, vol. 6, no. c, pp. 40547-40558, 2018.
[11] M. Lahby, L. Cherkaoui, and A. Adib, “An enhancedTOPSIS based network selection technique for next generation wireless networks," 2013 20th Int. Conf. Telecommun. ICT 2013, pp. 1-5, 2013.

[12] H. T. Yew, C. S. Kheau, R. K. Y. Chin, A. Chekima, and M. H. Satria, "Improved-TOPSIS based handover scheme for telemedicine service using heterogeneous wireless networks," in Proceedings - 2017 IEEE 2nd International Conference on Automatic Control and Intelligent Systems, I2CACIS 2017, 2017, vol. 2017-Decem, no. October, pp. 155-160.

[13] A. Sekkaki, "Optimal Vertical Handover based on TOPSIS algorithm and Utility function In Heterogeneous Wireless Networks," in 2017 International Symposium on Networks, Computers and Communications (ISNCC), 2017, pp. 1-6.

[14] M. A. Ben-Mubarak, B. M. Ali, N. K. Noordin, A. Ismail, and C. K. Ng, "Hybrid AHP and TOPSIS Methods Based Cell Selection (HATCS) Scheme for Mobile WiMAX," Sel. Top. WiMAX, 2013.

[15] S. Baghla and S. Bansal, "Effect of normalization techniques in VIKOR method for network selection in heterogeneous networks," 2014 IEEE International Conference on Computational Intelligence and Computing Research, IEEE ICCIC 2014. 2015.

[16] A. Kovac, M. Halas, M. Orgon, and M. Voznak, "Emodel Mos estimate improvement through jitter buffer packet loss modelling," Adv. Electr. Electron. Eng., vol. 9, no. 5, pp. 233-242, 2011. 